Supporting Information

\title{
Self-Powered Tactile Sensor with Learning and
}

\section{Memory}

Chaoxing Wu1,4, Tae Whan Kim', Jae Hyeon Park', Bonmin Koo', Sihyun Sung ${ }^{1}$, Jiajia Shao', Chi Zhang², and Zhong Lin Wang2,3*

1Department of Electronic and Computer Engineering, Hanyang University, Seoul 04763, Republic of Korea

2Beijing Institute of Nanoenergy and Nanosystems, Chinese Academy of Science, and National Center for Nanoscience and Technology (NCNST), Beijing 100083, People's Republic of China

${ }^{3}$ School of Materials Science and Engineering, Georgia Institute of Technology, Atlanta, Georgia 30332, United States of America

${ }^{4}$ College of Physics and Information Engineering, Fuzhou University, Fuzhou, 35000, PR China. 

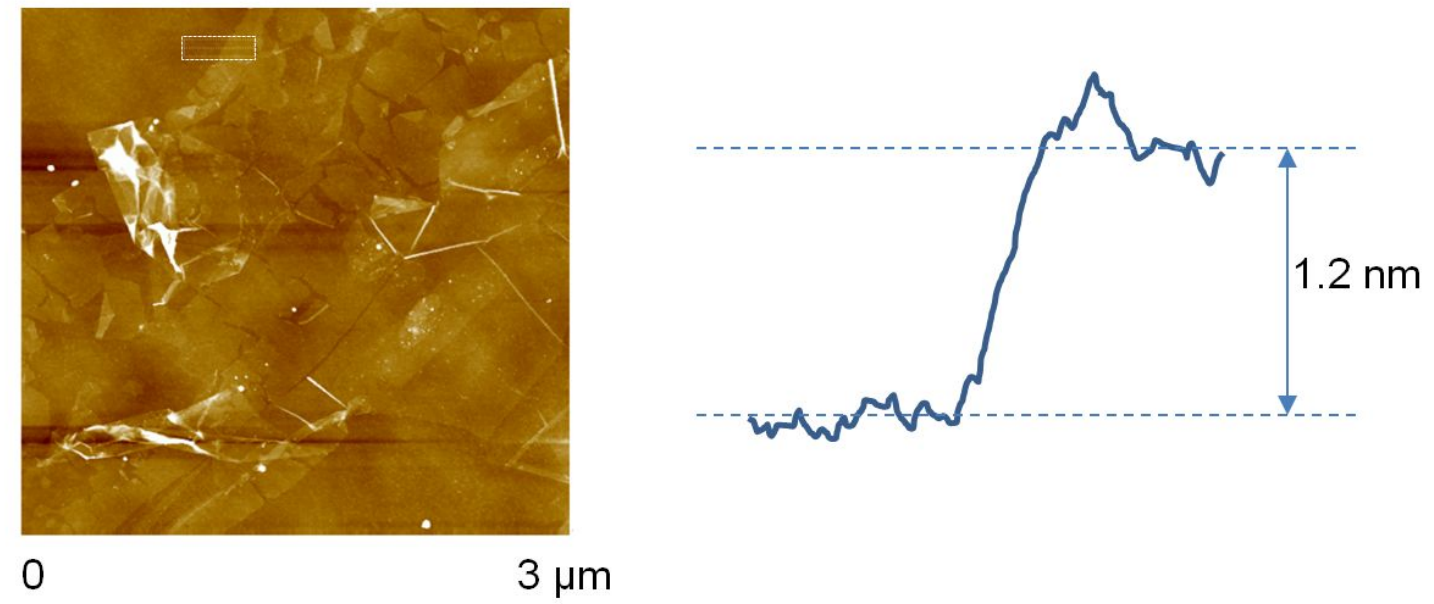

Figure S1. AFM image of the as-prepared rGO. The thickness of the rGO is about $1.2 \mathrm{~nm}$. 


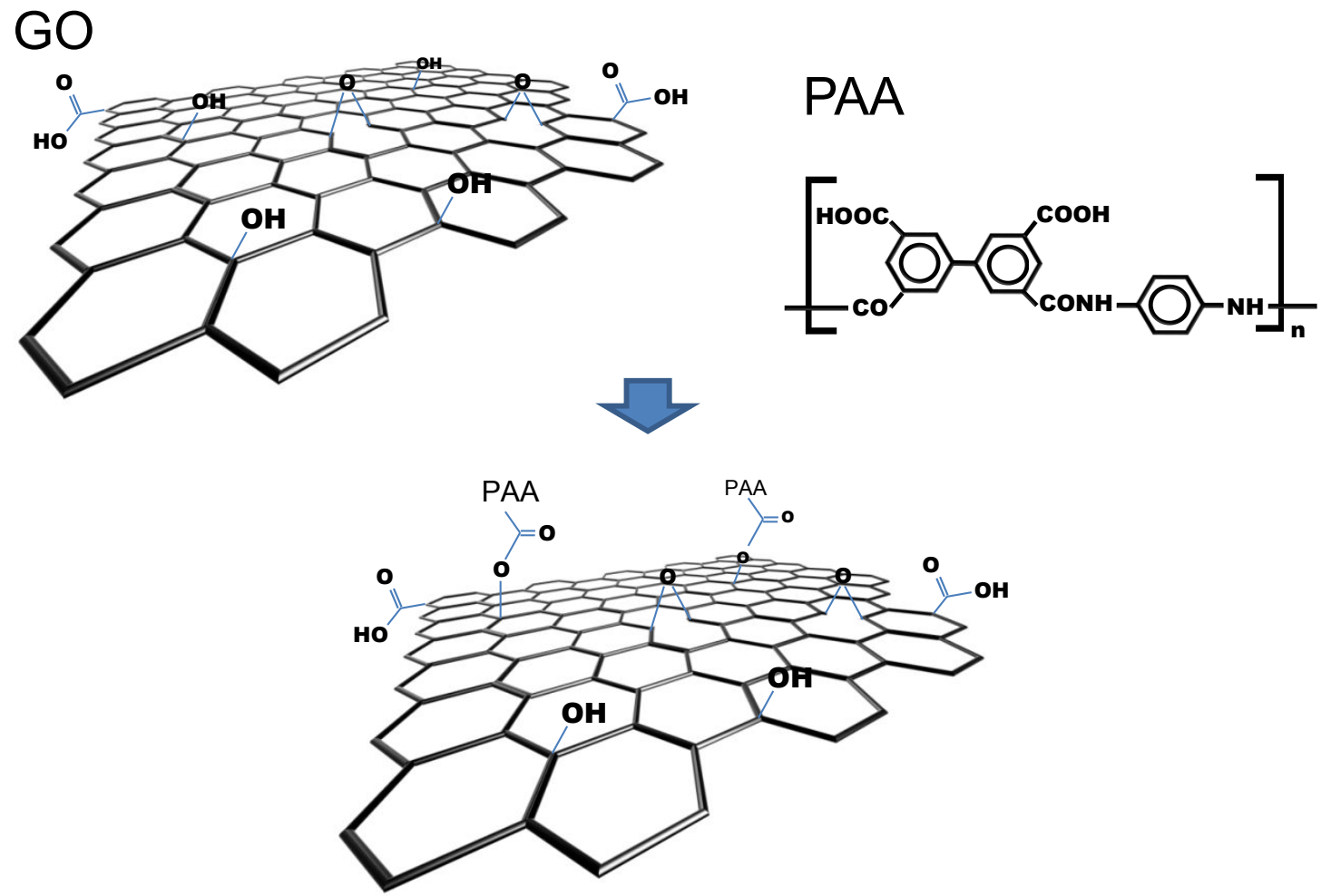

Figure S2. Schematic of GO sheet coupled with PAA. 


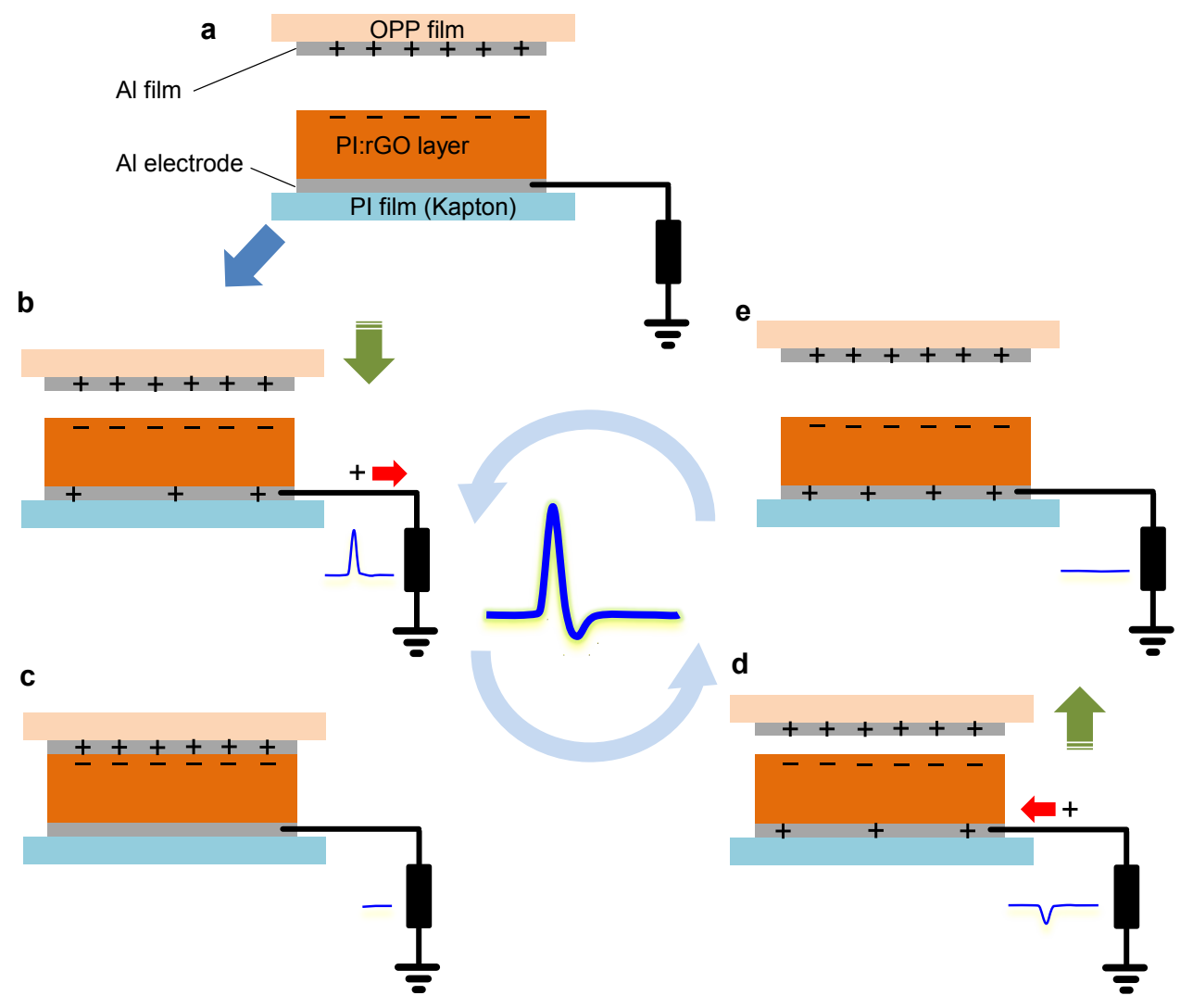

Figure S3. The basic working principle of the SE-TENG. (a)When the Al film comes into contact with the PI:rGO film under external pressure, electrons will transfer from the Al film to the PI:rGO surface due to the triboelectrification effect, leaving the PI:rGO film with negative charges and the Al film with an equal amount of positive charges. The charges are able to remain on the surfaces of the PI:rGO and the $\mathrm{Al}$ films for a period of time. (b),(c) When the $\mathrm{Al}$ is driven by an external force into contact with the PI:rGO film, a current flowing from the Al electrode to ground can be induced due to electrostatic induction. (d),(e) When the applied force is released, the $\mathrm{Al}$ moves away from the PI:rGO film, and a current with the opposite polarity is generated. 


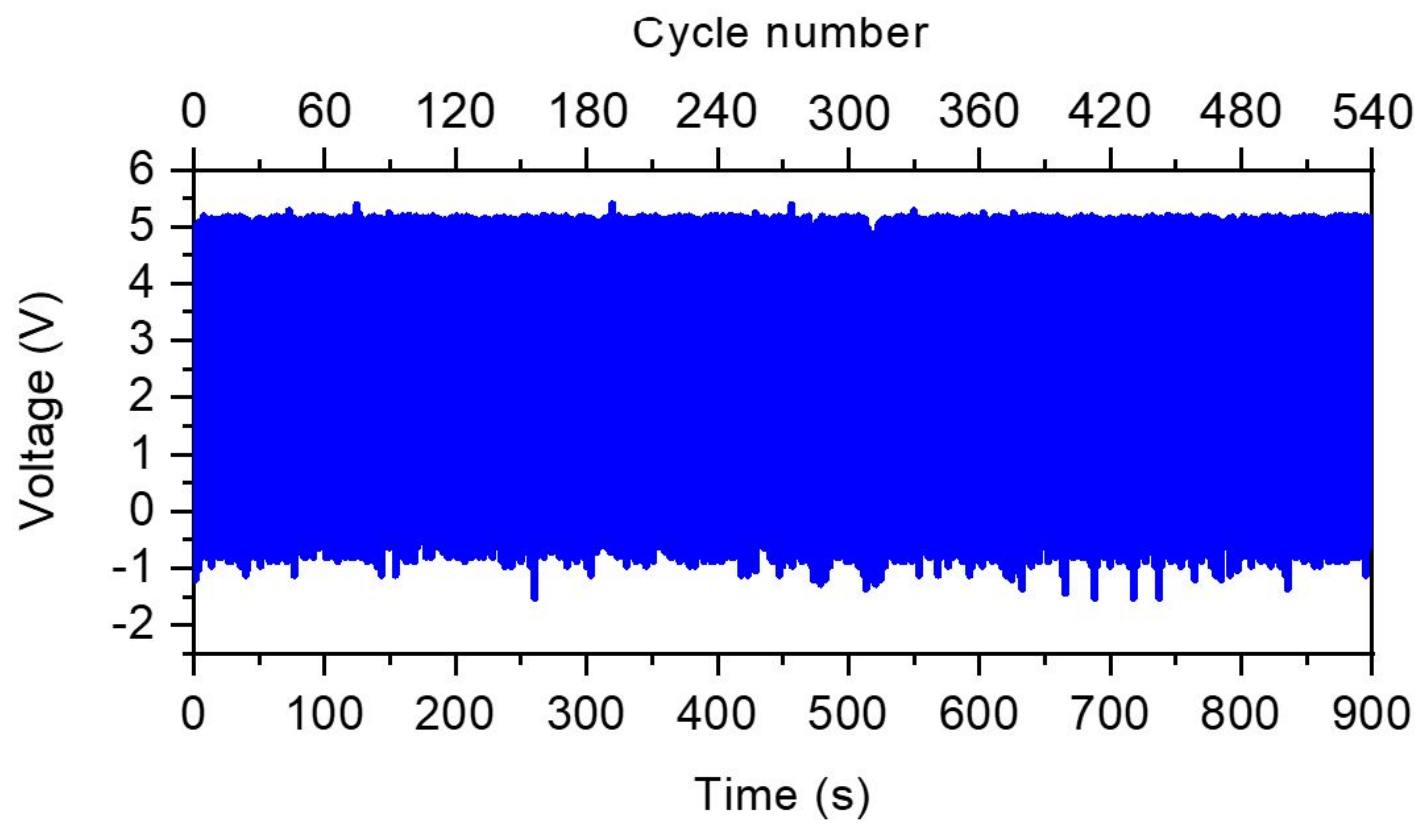

Figure S4. Durability of the device. When the output of the tactile sensor reaches a saturated value, more than 500 cycles of press-release are applied to the device. The output voltages are stable during the measurement. 
a

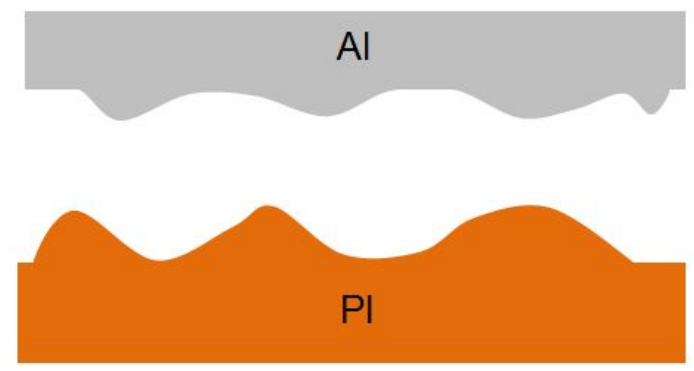

d

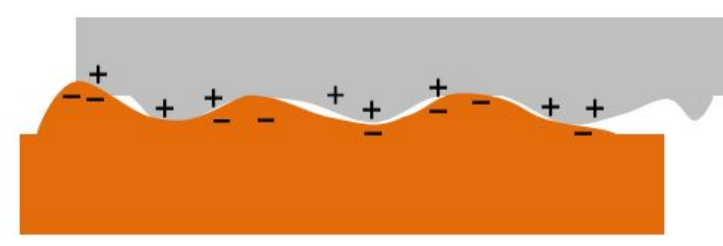

b
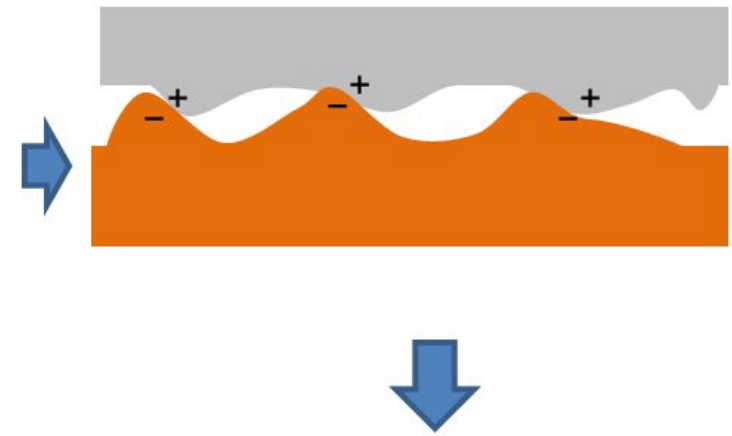

C
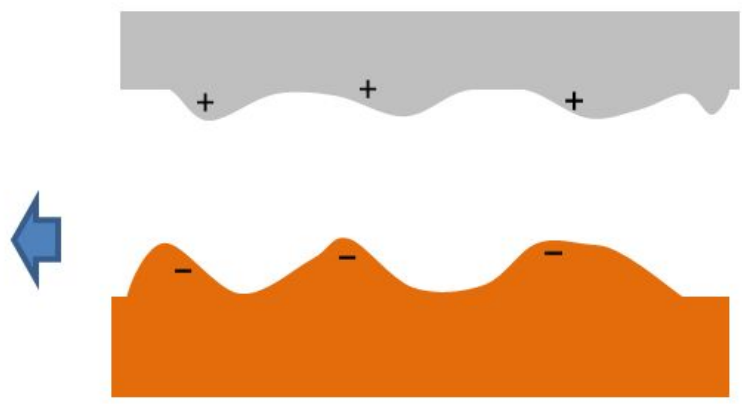

Figure S5. Schematic illustration of the triboelectrification between the Al and the PI films. (a) The Al and the PI films have microcosmic surface roughness. (b, c) During the first contact process, electrons transfer from the Al film to the PI film only at the local area of contact. The static electrons can remain in that area of the PI film due to its insulating property. (d) During the second contact process, the microcosmic contact area is inevitably changed. Thus, additional electrons can transfer from the Al film to another contact area on the PI film. As a result, the total number of static electrons on the surface of the PI film is increased. As the number of contact/release cycles increases, static electrons can increasingly accumulate on the surface of the PI film, and the output voltage increases accordingly. 

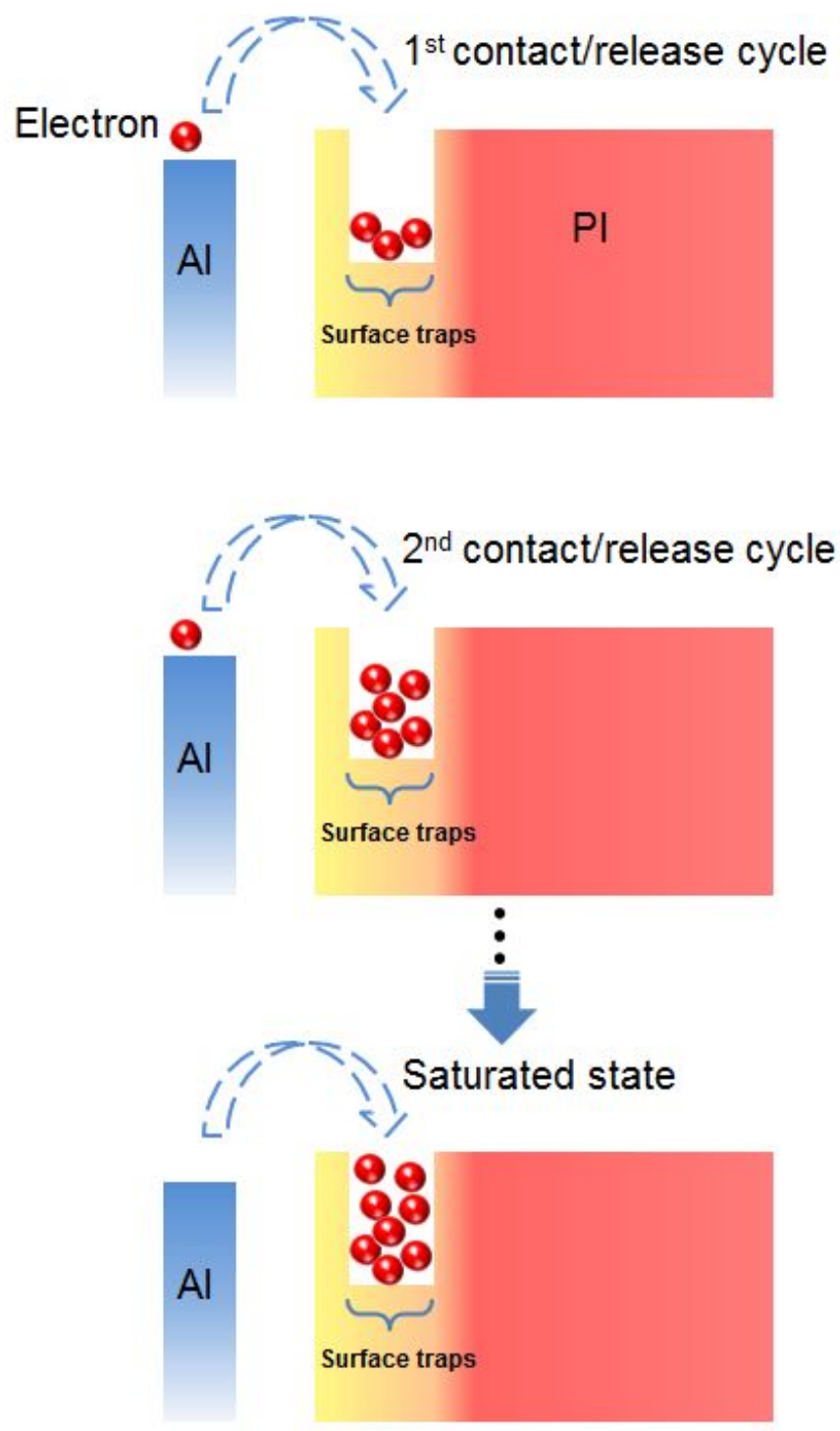

Figure S6. Schematic process showing the triboelectric electrons transferring from Al to the surface traps in the PI film. According to the surface states model, when a metal is in contact with a dielectric, those electrons with high energy in the metal hop up into the surface states of the dielectric. Thus, we can refer to these surface states as surface traps for static electrons. In the first contact-release cycle, when the Al film is in contact with the PI layer, electrons in the Al film hop up into the surface traps of the PI. Then, the occupied energy levels in the surface trap increase to the Fermi level of $\mathrm{Al}$, and the triboelectrification process stops. However, because of the microcosmic surface roughness of the Al and PI, only some of the surface traps are filled by the electrons. In the second training, a similar transfer of electrons from the Al layer to the PI layer occurs. As a result, the total number of generated triboelectric electrons increases, and the output voltage is definitely higher than the initial output voltage. After enough training, the triboelectric electrons captured in the PI surface traps reach a 
maximum, and the output voltage becomes saturated. Because the total number of surface traps in the PI film is limited, the final saturated electron density is only slightly higher than it is in the initial state, which is different from situation shown in Figure 2d. 
a

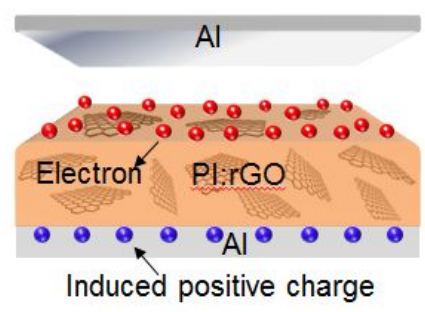

b

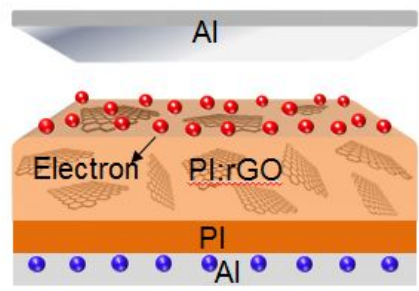

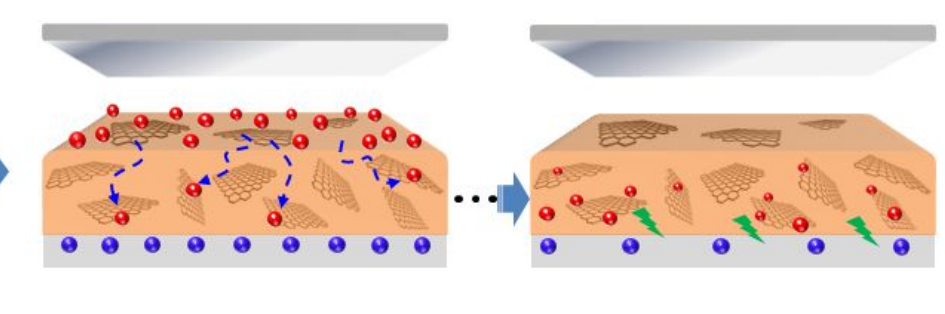

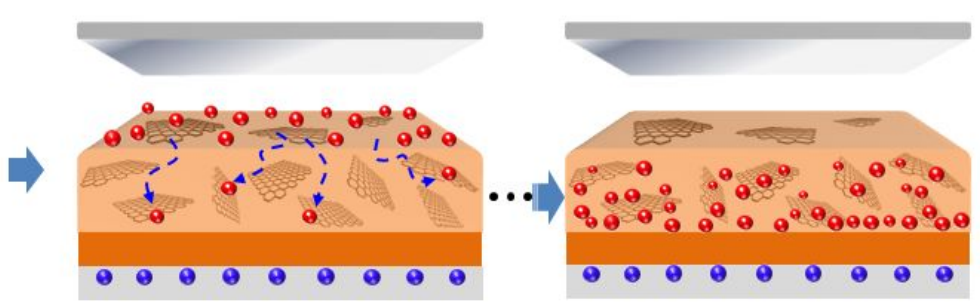

Figure S7. Schematic process showing the triboelectric electrons transferring in the negative friction layer. The triboelectrification process can be further divided into three subprocesses: the generation of triboelectric electrons, the storage of triboelectric electrons, and the loss of triboelectric electrons. The process of triboelectric electron loss decides the memory retention time. An electric field with a vertically upward direction will be set up between the contact PI:rGO surface and the Al electrode. Therefore, two electron-transfer modes exist: a drift process caused by the electric field and a diffusion process caused by the concentration gradient of electrons. For the PI:rGO layer, the drift and the diffusion processes will lead to a combination of triboelectric electrons with induced positive charges on the electrode, as shown in (a). However, for the PI:rGO/PI stacked structure, the insertion of a highly insulating PI layer can suppress the combination process and increase the retention time, as shown in (b). 


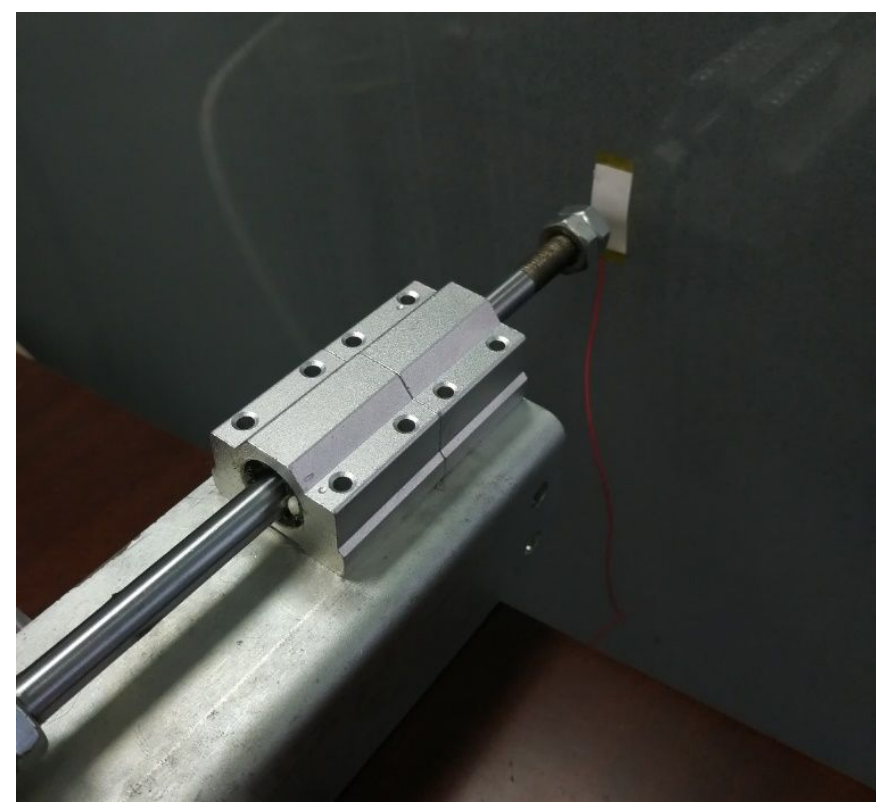

Figure S8. Measurement setup for the devices. Intelligent neuromorphic sensor is fixed on a fixed rigid substrate, and a linear motor is used to apply pressure to the device. 